\title{
The impact of COVID-19 on rare and complex connective tissue diseases: the experience of ERN ReCONNET
}

\author{
Rosaria Talarico, Silvia Aguilera, Tobias Alexander (D, Zahir Amoura, \\ Ana M. Antunes, Laurent Arnaud (D, Tadej Avcin, Lorenzo Beretta, \\ Stefano Bombardieri, Gerd R. Burmester, Sara Cannizzo, Lorenzo Cavagna, \\ Benjamin Chaigne, Alain Cornet, Nathalie Costedoat-Chalumeau, \\ Andrea Doria, Alessandro Ferraris, Rebecca Fischer-Betz, João E. Fonseca, \\ Charissa Frank, Andrea Gaglioti, Ilaria Galetti, Jürgen Grunert, \\ Vera Guimarães, Eric Hachulla, Frederic Houssiau, Luca Iaccarino, \\ Thomas Krieg, Marteen Limper, Fransiska Malfait @, Xavier Mariette, \\ Diana Marinello (D, Thierry Martin, Lisa Matthews, Marco Matucci-Cerinic, \\ Alain Meyer, Carlomaurizio Montecucco, Luc Mouthon, Ulf Müller-Ladner, \\ Simona Rednic, Vasco C. Romão, Matthias Schneider, Vanessa Smith, \\ Alberto Sulli, Farah Tamirou, Domenica Taruscio, Anna V. Taulaigo, \\ Enrique Terol, Angela Tincani, Simone Ticciati, Giuseppe Turchetti, \\ P. Martin van Hagen, Jacob M. van Laar, Ana Vieira, \\ Jeska K. de Vries-Bouwstra, Maurizio Cutolo and Marta Mosca
}

Abstract | During the COVID-19 pandemic, the need to provide high-level care for a large number of patients with COVID-19 has affected resourcing for, and limited the routine care of, all other conditions. The impact of this health emergency is particularly relevant in the rare connective tissue diseases (rCTDs) communities, as discussed in this Perspective article by the multi-stakeholder European Reference Network on Rare and Complex Connective Tissue and Musculoskeletal Diseases (ERN ReCONNET). The clinical, organizational and health economic challenges faced by health-care providers, institutions, patients and their families during the SARS-CoV-2 outbreak have demonstrated the importance of ensuring continuity of care in the management of rCTDs, including adequate diagnostics and monitoring protocols, and highlighted the need for a structured emergency strategy. The vulnerability of patients with rCTDs needs to be taken into account when planning future health policies, in preparation for not only the post-COVID era, but also any possible new health emergencies.

COVID-19 is an infectious disease caused by SARS-CoV-2, a newly discovered coronavirus. In March 2020, the WHO declared the COVID-19 outbreak to be a pandemic and as of 17 November 2020, the WHO had reported more than 54 million confirmed cases and more than 1 million confirmed deaths attributable to the disease ${ }^{1}$.

The COVID-19 pandemic has had a considerable effect on different aspects of rare and complex diseases, first on the risk of SARS-CoV-2 infection for people affected by these conditions, and also on the management of care and treatment during (and after) this global health emergency. The preliminary results of a Europe-wide survey organized by Rare Barometer (an initiative of the European Organisation for Rare Diseases), which was aimed at collecting the opinions, experiences and needs of patients with rare diseases, highlighted the many challenges faced by these patients as well as their families and caregivers during the pandemic ${ }^{2}$, including the interruption of routine medical care and rehabilitation therapies as well as many psychological barriers related both to isolation and to fear of infection with SARS-CoV-2 when receiving care in hospitals.

These same challenges can be readily related to autoimmune diseases, in which the rate and mechanisms of COVID-19 transmission have raised many concerns among scientists and patients, especially considering the well-known susceptibility of these patients to infections owing to their altered immune systems and to the use of immunosuppressive therapies ${ }^{3,4}$.

In parallel, owing to the so-called deleterious 'cytokine storm' that might explain the rapid worsening of some patients with COVID-19, some of the most common DMARDs, such as hydroxychloroquine, baricitinib, corticosteroids and tocilizumab, have been used in clinical trials for patients with COVID-19 who have hyperinflammation ${ }^{5,6}$. Thus, clinicians caring for patients with rheumatic diseases, as specialists in inflammation and the use of these drugs, have naturally been at the front line in treating COVID-19 in many cases. The interest in DMARDs has indeed helped to elucidate some of the pathogenetic mechanisms underlying COVID-19 $9^{7-9}$, but it has also caused tensions and, in some cases, shortages in the supply of some DMARDs (in particular hydroxychloroquine) $)^{10,11}$.

COVID-19 has also affected the management and treatment of patients with rheumatic and autoimmune diseases during the COVID-19 emergency. Publications reporting these effects have described challenges to provision of care, management of clinical trials and the psychological state of patients and their carers ${ }^{12,13}$.

These challenges are particularly relevant in the case of rare and complex connective tissue and musculoskeletal diseases (rCTDs), in which knowledge related to diagnosis, treatment and complications is often limited and expertise is scattered. rCTDs comprise a large number of diseases and syndromes, including hereditary conditions, rare systemic autoimmune diseases and complex 
systemic autoimmune diseases, and are characterized by the complexity of their rare and diverse clinical phenotypes (for example, neurological involvement in systemic lupus erythematosus, or cryoglobulinaemia or lymphoma in Sjögren syndrome). rCTDs have a tremendous impact on the health and well-being of patients around the world; they affect individuals by limiting their activities, affect societies by contributing to health-care costs, work loss, disability pensions, early retirement and the need for social support, and confer a considerable burden in relation to morbidity and mortality ${ }^{14,15}$.

In order to address considerations specific to rare and complex diseases, the EC (European Commission) has promoted the creation of 24 European Reference Networks (ERNs; see related links); the ERN on Rare and Complex Connective Tissue and Musculoskeletal Diseases (ERN ReCONNET; see related links) is aimed at improving the management of rCTDs including antiphospholipid syndrome, Ehlers-Danlos syndromes, idiopathic inflammatory myopathies, IgG4-related diseases, mixed connective tissue diseases, relapsing polychondritis, Sjögren syndrome, SLE, systemic sclerosis and undifferentiated connective tissue diseases across the EU (European Union).

The ERN ReCONNET's network of health-care providers (HCPs) currently comprises 26 full members from eight EU countries: Belgium, France, Germany, Italy, Netherlands, Portugal, Romania and Slovenia, and 13 affiliated partners from an additional 10 countries. This Perspective article presents the point of view of the ERN ReCONNET and was conceived and authored by ERN ReCONNET HCP representatives, European Patient Advocacy Group (ePAG) representatives, members of the steering committee and of the external scientific advisory board as well as representatives of the EC. Considering the complexity of the COVID-19 pandemic, in this article we aim to assess the effects of the health emergency on rCTD communities during the initial phase of the pandemic, bringing together input from multiple stakeholders within the ERN ReCONNET, representing patients, families, caregivers, expert clinicians, researchers and health economists. Moreover, we also discuss organizational and health economic considerations related to rCTDs in the context of COVID-19, which could be useful for the design of specific health-care plans in the context of possible future health emergencies. Finally, we outline the support provided by ERN
ReCONNET and other institutions during the first phase of the pandemic, as well as introducing possible contributions that ERN ReCONNET could provide in the future phases of managing COVID-19.

\section{Effects of COVID-19 on rCTD care}

The COVID-19 pandemic and the health emergency it has given rise to have had a substantial effect on numerous dimensions of health care, especially in the countries where the outbreak first emerged, which for Europe was Italy and Spain. Hospitals and HCPs had to face the unprecedented challenge of fighting against an unknown virus while caring for a tremendous number of patients with COVID-19 in need of high-level care. Consequently, there was a dire need to concentrate resources for the care of patients with COVID-19, thus affecting the resources dedicated to the management of all the other conditions and limiting the performance of routine care. The impact of the COVID-19 crisis on health care as well as on patients, families and caregivers was particularly relevant in the rCTDs communities, in which the main challenges were related to the clinical and organizational aspects of care ${ }^{10,16-18}$. In the following paragraphs, we discuss the main effects of COVID-19 on rCTDs from the perspective of clinicians, patients, health economists and institutions, all of whom are included in the ERN Re CONNET.

\section{Effects on clinical management}

Registries of patients with rCTDs and

COVID-19. During the initial phase of the pandemic, more specifically during the first months of 2020, the effect of SARS-CoV-2 on patients with rCTDs became an important clinical concern. Specifically, this concern focused on two essential clinical questions: are patients with rCTDs at a higher risk of contracting SARS-CoV-2, and, if they are infected, do they have a worse prognosis than those without rCTDs? In order to collect the evidence needed to answer these questions, several initiatives were launched across the globe, including the COVID-19 Global Rheumatology Alliance (REF. ${ }^{19}$ and related links) and the EULAR COVID-19 Rheumatology Database (see related links), as well as national registries that are collecting data of patients with rheumatological diseases and SARS-CoV-2 infection, such as the registry launched by the Italian Society of Rheumatology $y^{20}$. Preliminary data from these registries suggest that use of conventional DMARDs (including hydroxychloroquine) is neutral, chronic use of corticosteroids at doses above $10 \mathrm{mg}$ per day is deleterious and the use of biologics (especially TNF inhibitors) could be protective ${ }^{21,22}$. Moreover, data from the newly established registries also demonstrate that paediatric patients with rCTDs are at a much lower risk of acquiring clinically significant COVID-19 infection than patients over 61 years old ${ }^{23}$.

\section{Recommendations for the management of rCTDs in the context of COVID-19. For} the rheumatology community, EULAR has made a notable contribution with the publication of provisional recommendations for the management of rheumatic and musculoskeletal diseases (including most of the diseases covered by the ERN ReCONNET) in the context of SARSCoV-2 infection ${ }^{24}$. The document provides useful overarching principles, including the fact that so far there is no evidence that rheumatic patients are at a higher risk of contracting SARS-CoV-2 or of having a worse prognosis once infected. In addition, the recommendations state that the treatment plan should be continued in rheumatic patients who do not have suspected or confirmed diagnosis of COVID-19 and that the postponement of face-to-face rheumatological consultations should be considered on the basis of stability of the disease and of the tolerability of the treatments. Useful considerations were also provided by the World Scleroderma Foundation and answered the main clinical questions regarding SARS-CoV-2 infection and patients with $\mathrm{SSc}^{25}$.

\section{The effects of the COVID-19 pandemic} within ERN ReCONNET. Through internal discussions, the ERN ReCONNET has identified and prioritized a number of clinical and organizational challenges (FIG. 1). Specifically, patients with rCTDs and the health-care professionals treating them have experienced numerous clinical challenges during the pandemic, most frequently limitations to completing the diagnostic process and performing routine follow-up and monitoring. These challenges arose mainly from the limitations imposed by the measures necessary for prevention and control of SARS-CoV-2 transmission, including the closure of some clinics and/or the reorganization of services, which impeded face-to-face consultations. These limitations have often delayed the definition of the most appropriate treatment plans based on the diagnosis and on the disease activity of patients with rCTDs. As reported by the ERN ReCONNET HCPs, in order 
to avoid the risk of contagion among patients in the hospital setting as much as possible, clinicians have also opted - when considered appropriate - to switch from infusion to subcutaneous therapies, so that patients could reduce their visits to the hospital.

Still to be clarified, among many other issues, is whether the process for the screening of patients prior to commencement of immunosuppressive therapy should be updated, taking into consideration the possibility of a positive test for SARS-CoV-2 infection (by swab and/or serology). In particular, it remains to be confirmed whether systematic SARS-CoV-2 screening needs to be incorporated into existing protocols, in order to avoid the hazardous consequences of starting a new biologic therapy in patients with undisclosed infection ${ }^{8}$. From the above considerations, it seems clear that the pandemic has had a considerable effect on the clinical management of rCTDs and that its influence will continue in the coming months and even years. The period of disruption will probably be prolonged both because the pandemic is continuing to affect health-care systems at present, and subsequently patients with rCTDs might still encounter barriers and challenges to the provision of the care they need. For these reasons, the care of patients with rCTDs during the post-pandemic era should be carefully planned and implemented, profiting from the experience gained during the health emergency.

\section{Effect on organization of care}

The pandemic has put HCPs under enormous pressure, and has made health-system collapse a real risk, owing to the need to provide care for patients affected by COVID-19. As the ERN ReCONNET $\mathrm{HCP}$ and ePAG representatives can attest, health-care professionals treating patients with rCTDs have faced numerous organizational challenges during the pandemic (FIG. 2).

Heads of units, hospital managers and health-care professionals have joined forces in order to reorganize the services of whole hospitals, to ensure adequate resources for COVID-19 units while maintaining, when and if possible, essential health services for patients with rCTDs. Many ERN ReCONNET HCPs had to cope with the fact that some of their staff were reassigned to COVID units and in some cases units treating patients with rCTDs were even converted into COVID units; they have also faced shortages and tensions in the

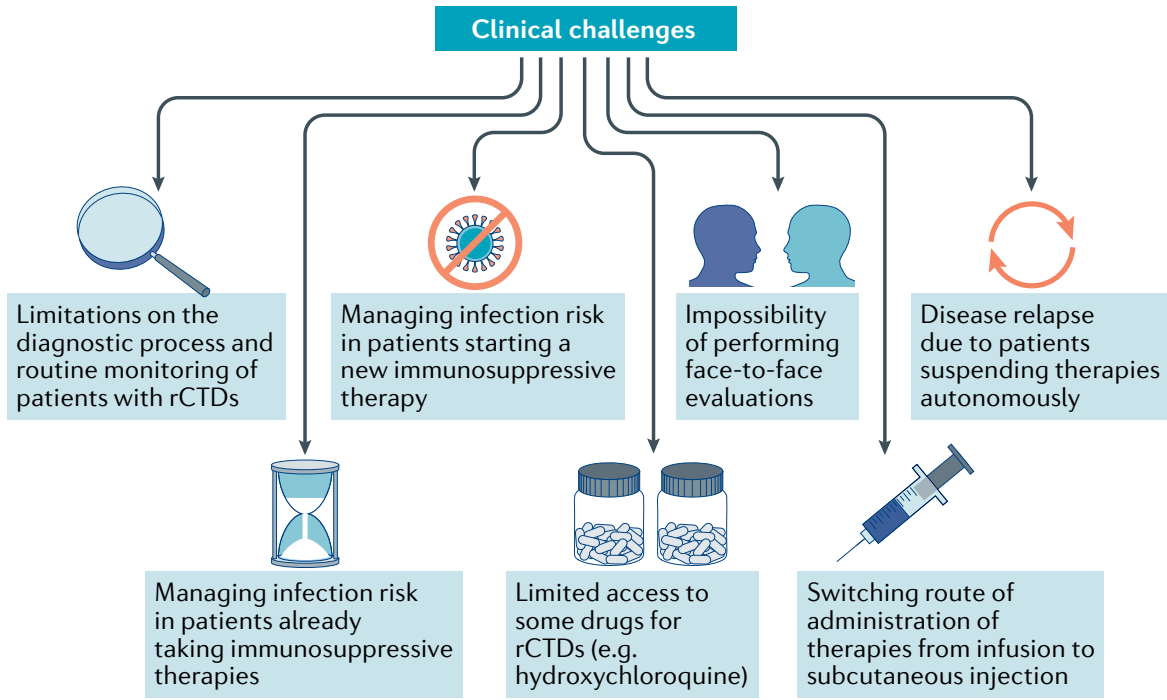

Fig. 1 | Clinical challenges faced by patients and health-care providers during the COVID-19 pandemic. Discussions within the European Reference Network on Rare and Complex Connective Tissue and Musculoskeletal Diseases (ERN ReCONNET) have identified a number of clinical challenges faced by patients with rare connective tissue diseases (rCTDs) and the health-care professionals treating them during the COVID-19 pandemic. Some concerns relate to the fear and/or consequences of these patients contracting COVID-19, whereas others reflect changes to clinical protocols implemented to prevent or control SARS-CoV-2 transmission in clinics.

provision of medicines and of personal protective equipment. As the rate of new cases has slowed across Europe, in most rCTD units the staff have returned from COVID units, although patient flow for inpatient and outpatient activities has had to be reorganized in order to enable social distancing and to limit the risk of contagion as much as possible. Many of these challenges are still being faced by rCTD units during the current second wave of the pandemic.

Organizational challenges caused by the pandemic have frequently interrupted the care provided to patients with rCTDs, including the suspension of inpatient and outpatient clinics, or provision of infusion therapies. ERN ReCONNET HCPs and ePAGs have also had to manage requests from a large number of patients for information on their risk of being infected because of their disease or to their treatments, and have needed to use telemedicine to provide routine services instead of face-to-face evaluations or to identify patients for whom direct assessments cannot be postponed ${ }^{26}$.

Although the ERNs are already quite experienced in terms of eHealth owing to the usage of the Clinical Patient Management System (CPMS; see related links), a web-based secure platform used by all ERNs for the virtual discussion of clinical cases across borders, the use of telemedicine in routine clinical practice is still being implemented in many health-care systems. However, the limitations imposed by the pandemic have enabled health-care professionals to activate, within a very short time frame, different initiatives related to telemedicine, such as telephone and video consultations and issuing prescriptions electronically. Many of these initiatives were also reimbursed by various health-care systems in Europe ${ }^{27,28}$. The use of telemedicine during the pandemic has revealed the potential of this approach in the routine follow-up of patients even beyond the pandemic; clearly, this experience could be translated into a wider, harmonized and systematic usage of telemedicine across Europe, benefiting patients, health-care professionals and institutions.

\section{Effects on patients with rCTDs}

Besides clinicians and health-care professionals, the COVID-19 pandemic has heavily affected the community of patients with rCTDs. The patient-centred approach of ERN ReCONNET has encouraged the ERN ReCONNET ePAG representatives to discuss together the effects of the pandemic on patients with rCTDs across Europe.

During the pandemic, patients affected by rCTDs have had to deal with many more complex situations than healthy people, owing to the fact that many of them are treated using immunosuppressive therapy and that lung involvement is a common manifestation in $\operatorname{rCTDs}^{11,29}$. The (in) 


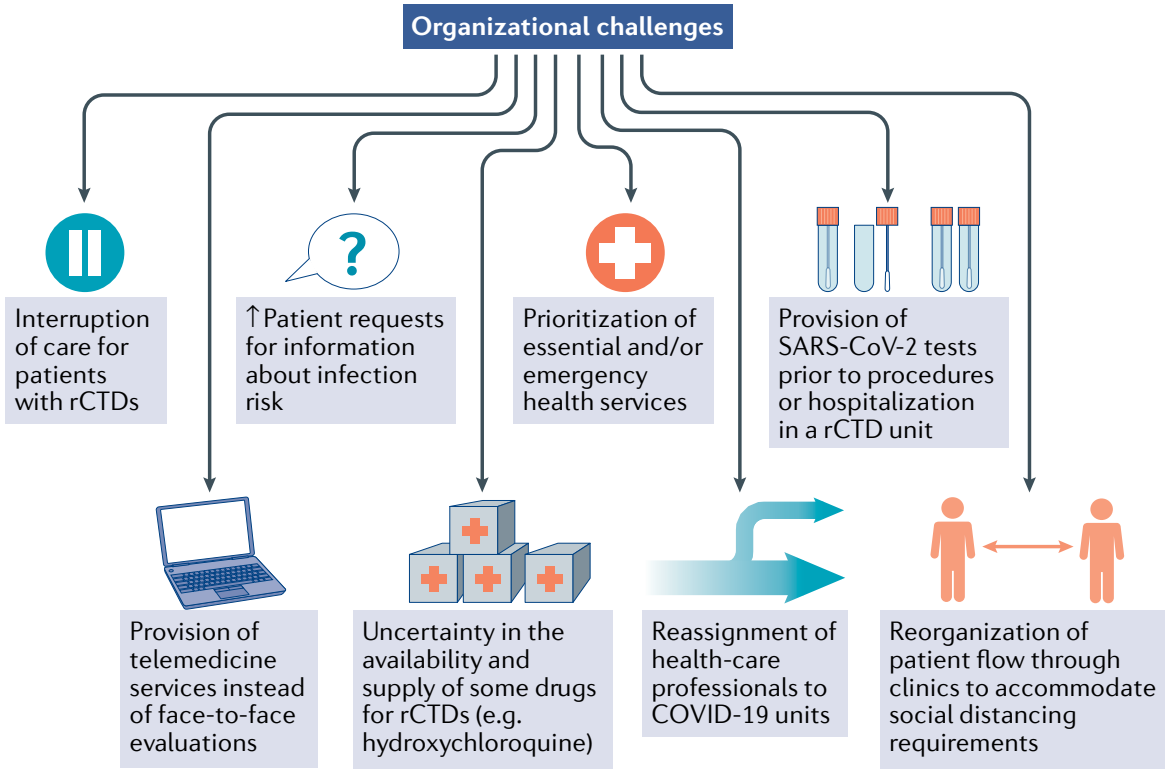

Fig. 2 | Organizational challenges faced by health-care clinics during the COVID-19 pandemic. Health-care systems are under sometimes severe strain owing to the need to care for patients with COVID-19, which has had a knock-on effect for the management of other patients, including those with rare connective tissue diseases (rCTDs). Health-care providers within the European Reference Network on Rare and Complex Connective Tissue and Musculoskeletal Diseases (ERN ReCONNET) have faced many organizational challenges during the pandemic, many of which have arisen as a consequence of restrictions imposed on clinics in order to prevent and control SARS-CoV-2 transmission.

voluntary lockdown also had a substantial influence on the quality of life and daily activities of these patients because of a shift to teleworking, the need to coordinate 24-h family life or the loss of fundamental support from caregivers, particularly for those patients living alone. At the same time, patient organizations (such as the Federation of European Scleroderma Associations (FESCA); see related links) had to suspend their routine activities while making great efforts to provide psychological support via telephone or online assistance. The challenges faced by patients with rCTDs might be easily related to the fact that patients affected by rCTDs are, even in routine scenarios, at an increased risk of infection and the fear of contagion has often increased the need for psychological support for patients with rCTDs. Patients experiencing isolation and fear have, in fact, expressed the need to maintain the psychological support service after the pandemic as well.

Moreover, as mentioned above, regular access to medical advice and continuity of care for non-COVID-19 patients have been disrupted as hospitals have become COVID-19 units and concerns for the safety of patients emerged. As mentioned above, the safety of patients with rCTD patients was, and still is, particularly important because of their vulnerability; protecting these patients from the risk of contagion should remain a priority. The use of different telemedicine tools, even though they have not always been part of an established system, has played a crucial role in compensating for the discontinuation of routine care, by providing an additional service for patients unable to attend hospital. Dispensation of hospital medications or treatments has been replaced by community pharmacy delivery or alternative treatment methods when possible, and access to emergency rooms has been regulated to avoid contact with potentially contagious patients. Post-COVID, maintaining the delivery of hospital medicines via community pharmacies, secure access to emergency rooms and the possibility of at-home management of therapies is anticipated to have a positive impact on the quality of life and on the economic burden of patients. A CPMS-like teleconsultation platform should be developed to enable interactions between patients and clinicians and guarantee safe data management; by doing this, the lessons learned during the pandemic would deliver a positive benefit for patients.

Another important consideration for patients with rCTDs during the pandemic is related to the fact that some rCTDs are treated with hydroxychloroquine. Reports of the potential of hydroxychloroquine ${ }^{30}$ as a treatment for COVID-19 (REF. ${ }^{7}$ ) created an increased demand that led to supply gaps in many countries, causing anxiety and fear among patients who rely on the drug. A survey performed in one European rCTD community highlighted that only $44 \%$ of patients with 'on label' hydroxychloroquine use obtained on-the-spot delivery of their drug in early April, and 6.7\% experienced a delay of 2 weeks or more ${ }^{31}$. ERN ReCONNET ePAGs have actively engaged with several patient groups to monitor the availability of the drug, to monitor the prescription and distribution rules in different countries, and to obtain emergency supply routes direct from the manufacturer, in order to assist and reassure the patient community.

\section{Organizational considerations}

The COVID-19 pandemic has raised a number of organizational and health economic considerations related to the management of rCTDs. Pertinent questions are summarized in BOX 1 and potential solutions are discussed in this section.

\section{Ensuring continuity of care}

The challenges and risk of health-care system collapse experienced during the COVID-19 pandemic have highlighted, among other things, how essential it is to maintain continuity in the provision of care, especially for the management of rare diseases. Interruption of the care provided to patients with rCTDs and other rare diseases was in fact one of the most frequent health problems experienced by patients and health-care professionals. For this reason, it is worth considering whether potential future emergencies could threaten health-care systems and services and challenge the maintenance of adequate care. From an organizational point of view, the COVID-19 outbreak challenged coordination strategies, making it difficult to balance the need for a rapid response to COVID-19 while ensuring provision of essential health services to patients with rCTDs. As suggested in the preliminary operational guidance document developed by the $\mathrm{WHO}^{32}$, the identification of context-relevant essential services is crucial to prioritizing and maintaining care delivery during an outbreak, with, for example, the creation of disease-specific structured care pathways and well-designed patient flow (that is, screening, triage and targeted referral). This guidance 
provides operational suggestions that could represent the basis for emergency strategies if applied and adopted in the arena of rare and complex diseases and supported by the experience, expertise and infrastructures of the ERNs. Addressing the challenges associated with maintaining essential health services with the support of ERN ReCONNET could greatly benefit rCTD communities, by enabling the needs specific to rCTDs and by considering and implementing the priorities of clinicians, hospital managers, patients and other stakeholders in future plans.

\section{Integrating eHealth tools}

The limitation of face-to-face consultations enabled the widespread application of eHealth and telemedicine tools, such as video and telephone consultations. During the COVID-19 health emergency, eHealth and telemedicine have been and will continue to be valuable tools for monitoring patients with rare and complex diseases in order to identify and prioritize patients needing immediate care. Although face-to-face evaluation and clinical examination remain essential in the management of rCTDs, eHealth and telemedicine tools could facilitate optimization of care (for example, improving the monitoring of adherence to treatment, self-monitoring of disease activity, enabling communication with general practitioners, etc.), especially if integrated with medical devices such as apps for mobile phones and wearable devices. In order to implement effective and appropriate eHealth and telemedicine tools, EU-wide initiatives could be developed not only to create tools to be used across different rare diseases, but also to ensure their usage in different (both emergency and routine) health-care scenarios. In addition, existing EU reimbursement schemes could be adapted and adopted to ensure the recognition of these crucial services across Europe. In this regard, the experience and knowledge gained in recent years by the ERN ReCONNET might be particularly precious in the planning of future eHealth and telemedicine initiatives and policies for rCTDs, especially considering that the ERNs represent a model to ensure adequate and equitable access to care and treatment across different countries, mainly by sharing the knowledge and expertise of their members and patient representatives.

\section{Planning for future emergencies}

The unprecedented emergency precipitated by the COVID-19 pandemic has highlighted the strengths and weaknesses of our organizations and of our health-care policies. During an emergency, priorities necessarily - change. However, as discussed in this paper, this reprioritization risks under-diagnosis (or missed diagnoses), under-treatment or missed treatment, inadequate monitoring, or simply delays to health-care procedures. Preparation is required in order to avoid the negative health and economic implications for rCTDs arising from COVID-19 and potential future emergencies, which we suggest can be done along two directions. The first direction is to continue to increase the awareness of all the relevant stakeholders (policy-makers, hospital managers, national authorities, patients' organizations etc.) about the relevance of rCTDs and their health-related, organizational and economic burden. Rare and complex diseases, and rCTDs in particular, should always occupy an important place in the agenda of health-care policy makers, and the expense - continuous and systematic allocated to health care in this area should be considered an investment and not a $\operatorname{cost}^{33}$. The second direction is to invest in cross-border European infrastructure for the management of rare and complex diseases, which will already be in place for when emergencies happen. ERN ReCONNET, and ERNs in general, represent a common platform that can be relied on in both 'ordinary' and 'extraordinary' times to ensure that patients are not neglected, even when priorities are redefined during an emergency. Continued investment in and upgrading of these infrastructures by the EC and its member states will ensure that they are robust when new emergencies arise, thus helping to minimize the negative health, organizational and economic implications for the management of rare and complex diseases.

\section{Institutional support}

Institutions, including the ERNs, can provide support to clinicians and patients with rCTDs in the COVID-19 era in several different ways. In this section, we outline how the EC, ERNs (particularly ERN ReCONNET) and related organizations have responded to the pandemic and contributed to rCTD communities.

\section{The European Commission response}

Since the very beginning of the pandemic, the EC has been coordinating a common European response in order to reinforce public health sectors and mitigate the socioeconomic ramifications in the $\mathrm{EU}^{34}$. The EU response to the SARS-CoV-2 outbreak included closure of the EU's external borders to limit transmission of the virus, the introduction of 'green lanes' (border crossing routes established to ensure the free circulation of goods within the EU), the promotion of research into COVID-19 and granting access to the RescEU stockpile of medical equipment and the EU Solidarity Fund (see related links). Among the many initiatives promoted in the EU, the EC launched the COVID-19 Clinical Management Support System $(\mathrm{CMSS})^{35}$ in March 2020 as a tool to enable health-care professionals involved in handling the COVID-19 crisis across the EU (including the UK) and the European Economic Area to exchange information and consult with colleagues about the treatment and management of COVID-19, facilitate the clinical decision-making process and improve training, similar to the ERN approach. The CMSS consists of the COVID-19 Clinicians Network (a database of clinicians treating COVID-19), a central helpdesk and a web-conferencing system for online conferences and webinars ${ }^{35}$. So far, several health-care professionals from many HCPs have connected to the COVID-19 Clinicians Network. Virtual discussions are also taking place in the framework of the CMSS and training webinars are being organized on COVID-19 and rare and complex diseases. These initiatives clearly show how EU-wide initiatives can contribute to the improvement of care and knowledge, topics that are particularly important in the management of both COVID-19 and rCTDs.

\section{Box 1 | Organizational and health economic considerations}

The organizational and health economic challenges faced by health-care providers, institutions, patients and their families during the COVID-19 pandemic highlight important questions to be considered regarding the future management of rare connective tissue diseases (rCTDs).

- How should care be reorganized to guarantee continuity in the provision of care, especially for patients with rCTDs?

- How can eHealth and telemedicine be integrated into health-care systems following the experience of using these approaches during the COVID-19 pandemic?

- How can we prevent the negative health and economic implications of COVID-19 and future emergencies for the care of patients with rCTDs? 


\section{COVID-19 and the ERNs}

The know-how and experience of the ERNs with respect to sharing of knowledge, networking and education provided a valuable model for the development of the CMSS initiative. In particular, the implementation of the CPMS provided the background for the EC to develop and establish the CMSS and the related COVID-19 Clinicians Network.

Many ERNs have also supported the EC's Directorate-General for Health and Food Safety (DG SANTE; the department responsible for EU policy on food safety and health and for monitoring the implementation of related laws) with the organization of a series of free webinars on COVID-19, which are contributing expertise and knowledge gathered on COVID-19 and rare and complex diseases. The live webinars organized since April 2020 explore a range of themes across various diseases, such as the use of anti-epileptic drugs in patients with COVID-19, providing remote care for patients with rare bone diseases during the pandemic, COVID-19 and intensive care medicine, and the impact of COVID-19 on people living with a rare disease. In addition, most ERNs are also setting up targeted initiatives related to COVID-19 and their individual disease areas. All webinars are publicly available on the EC website, as is a regularly updated list of the various ERN initiatives $^{36}$.

\section{ERN ReCONNET initiatives}

Considering the substantial impact of the COVID-19 outbreak within rCTD communities and the need for reliable information on COVID-19 and rCTDs (amid 'fake news' and inaccurate sources), ERN ReCONNET has developed a series of relevant initiatives. Since the beginning of the pandemic, a specific section of the ERN ReCONNET website has been dedicated to the COVID-19 pandemic $^{37}$ in order to provide a comprehensive list of reliable and authoritative resources to inform and update patients and health-care professionals on COVID-19-related topics. This section includes extensive resources from organizations, including scientific societies (EULAR and national rheumatological societies), the EC and the European Organisation for Rare Diseases.

ERN ReCONNET has also endorsed recommendations for patients with rheumatic musculoskeletal diseases issued by $\mathrm{Fai}^{2} \mathrm{R}$ (see related links), the French health-care network for rare autoimmune and autoinflammatory diseases ${ }^{38}$. These recommendations emphasize the importance of continuing treatment plans and provide useful information on how to act in the case of contact with someone who has tested positive for SARS-CoV-2 or in the case of symptoms ascribable to the virus. Following the ERN ReCONNET's endorsement of the Fai ${ }^{2} \mathrm{R}$ recommendations, the ERN ReCONNET ePAGs have supported both the $\mathrm{Fai}^{2} \mathrm{R}$ and the ERN ReCONNET by providing translations of the documents issued by $\mathrm{Fai}^{2} \mathrm{R}$ ("Recommendations for patients suffering from rheumatic musculoskeletal diseases" and "How to live better during this containment period") in 16 different languages, which are published on the ERN ReCONNET website, together with the English and French versions developed by $\mathrm{Fai}^{2} \mathrm{R}^{39}$. Starting from the translations, the ERN ReCONNET also created infographics (also available in 16 languages) to be shared with the rCTD communities on social media platforms. This strong collaboration among ePAG representatives and their network of patients, the $\mathrm{Fai}^{2} \mathrm{R}$ and ERN ReCONNET demonstrates that joining efforts can really have a positive influence, especially on the lives of patients, for whom the translations provide access to useful information on COVID-19 and their disease in their own language.

The scarcity of information on rCTDs and COVID-19 has also motivated ERN ReCONNET to promote the organization of educational activities and dissemination of updates for patients and health-care professionals on these topics from the point of view of a European network ${ }^{40}$, including a series of webinars. The initiatives organized to date also serve to focus attention on rCTDs despite the pandemic, highlighting the added value of the ERN ReCONNET even during a global health emergency and representing good practice applicable outside the ERN scenario as well. Considering the acute need for guidance and specific information on COVID-19 and rCTDs, and that the ultimate goal of the ERN ReCONNET stakeholders and institutions is to improve the care of patients with rCTDs in Europe, more initiatives will be organized by ERN ReCONNET on these topics, both for patients and for health-care professionals, in order to provide a tangible contribution to rCTD communities.

\section{CNMR initiatives}

Another example of institutions providing a rapid and useful response to the COVID-19 pandemic is the Italian National Rare Diseases Centre (CNMR; see related links) formally established within the National
Institute of Health (Istituto Superiore di Sanità; ISS) in 2008; the CNMR is the central coordination body of the Italian national network for rare diseases. The CNMR carries out scientific research and public health activities related to rare diseases, at both the national and the international level.

Considering the high number of COVID-19 cases that occurred in Italy at the beginning of the outbreak in Europe ${ }^{41,42}$, the CNMR has worked intensively on a number of activities and initiatives related to COVID-19 and rare diseases. The CNMR formed a task force dedicated to rare diseases and COVID-19, involving experts in rare diseases both internal and external to the ISS. The task force has coordinated a series of weekly webinars ${ }^{43}$ dedicated to general public health issues related to the pandemic, as well as to specific aspects of rare diseases in relation to COVID-19, involving the WHO, national and regional institutions, CNMR centres of expertise, ERNs (including ERN ReCONNET, as well as ERNs for rare endocrine conditions (ENDO-ERN), rare haematological diseases (ERN-EuroBloodNet), rare neurological diseases (ERN-RND) and others), ePAGs and Italian patients' associations. The CNMR task force has also developed several guidance documents ${ }^{44}$ that provide recommendations and support for the management of several rare conditions during the COVID-19 pandemic, including Kawasaki disease and acute multisystem inflammatory syndrome in children and adolescents. In addition, the CNMR task force, together with the Italian federation of rare disease associations (UNIAMO), organized a national survey to explore the effects of COVID-19 on Italian patients with rare diseases, the results of which were presented in a webinar. The main difficulties faced by patients and their caregivers include problems regarding both health care and social health support during the pandemic, as well as a substantial unmet need for information. Most relevant, more than half of the survey's respondents reported challenges in accessing follow-up care.

\section{Conclusions}

The COVID-19 pandemic has brought many challenges to the already vulnerable communities of patients with rCTDs. However, many of these challenges can be translated into positive lessons to be applied in the post-COVID era.

One of the main positives to emerge from the pandemic is surely the use of eHealth and telemedicine in the provision of care to patients with rCTDs. The experience 
gained during the pandemic, building on the previous knowledge obtained through the use of CPMS by ERNs, has highlighted the need to integrate eHealth and telemedicine into routine clinical practice as a tool to improve and optimize the management of rCTDs. In many cases, the alternation of telemedicine and face-to-face evaluation could in fact be considered when disease activity is stable, enabling patients to avoid the unnecessary burden of travelling, as well as its related costs and social and work-related disadvantages. Therefore, it is desirable that telemedicine and eHealth should be taken into consideration in future health policies and planning, to enable their successful integration into the health-care services provided to patients with rCTDs. Importantly, such policies could also promote the optimization and implementation of consultation between general practitioners and clinicians caring for patients with rCTDs.

In addition, the clinical and organizational challenges faced during the pandemic demonstrate how important it is to ensure the continuity of adequate diagnostic and monitoring protocols in the management of rCTDs. At present, we do not know whether the pandemic and the related lockdown might have indirectly influenced the exacerbation of the disease in some patients with rCTDs or contributed to delays in diagnosis; only observation in the coming months is likely to provide potentially useful information. What we do know is that, because new health emergencies might occur in the future, a specific and structured emergency strategy could be developed for the management and care of rCTDs, as well as for other rare and complex conditions. The vulnerability and the specific needs of patients with rCTDs must, in fact, be taken into account when planning future health policies, in preparation for not only the post-COVID era, but also any possible new health emergencies. To this end, the contribution of ERNs to the development of such strategies could add important value, supporting scientific societies and policymakers in providing for the needs and priorities of the Europe-wide rCTD communities.

Rosaria Talarico', Silvia Aguilera², Tobias Alexander (iD ${ }^{3}$, Zahir Amoura ${ }^{4}$, Ana M. Antunes ${ }^{5}$, Laurent Arnaud (D) ${ }^{6}$ Tadej Avcin ${ }^{7}$, Lorenzo Beretta ${ }^{8}$, Stefano Bombardieri ${ }^{9}$, Gerd R. Burmester ${ }^{3}$, Sara Cannizzo ${ }^{1,10}$,

Lorenzo Cavagna ${ }^{11}$, Benjamin Chaigne ${ }^{12}$, Alain Cornet ${ }^{13}$, Nathalie Costedoat-Chalumeau ${ }^{12}$, Andrea Doria ${ }^{14}$, Alessandro Ferraris ${ }^{15}$, Rebecca Fischer-Betz ${ }^{16}$, João E. Fonseca ${ }^{17}$, Charissa Frank ${ }^{18}$, Andrea Gaglioti ${ }^{1}$, Ilaria Galetti19, Jürgen Grunert ${ }^{20}$, Vera Guimarães ${ }^{21}$,
Eric Hachulla ${ }^{22}$, Frederic Houssiau ${ }^{23}$

Luca laccarino ${ }^{14}$, Thomas Krieg ${ }^{24}$, Marteen Limper ${ }^{25}$, Fransiska Malfait iD ${ }^{26}$, Xavier Mariette ${ }^{27}$,

Diana Marinello iD ', Thierry Martin ${ }^{28}$, Lisa Matthews ${ }^{29}$, Marco Matucci-Cerinic ${ }^{30}$, Alain Meyer ${ }^{6}$, Carlomaurizio Montecucco" ${ }^{11}$, Luc Mouthon'2, Ulf Müller-Ladner ${ }^{31}$, Simona Rednic ${ }^{32}$, Vasco C. Romão ${ }^{17}$, Matthias Schneider ${ }^{16}$, Vanessa Smith ${ }^{33}$, Alberto Sulli ${ }^{34}$, Farah Tamirou ${ }^{23}$, Domenica Taruscio ${ }^{35}$, Anna V. Taulaigo ${ }^{5}$, Enrique Terol ${ }^{36}$, Angela Tincani ${ }^{37}$, Simone Ticciati' ${ }^{1}$, Giuseppe Turchetti ${ }^{10}$, P. Martin van Hagen ${ }^{38}$, Jacob M. van Laar ${ }^{25}$, Ana Vieira ${ }^{39}$, Jeska K. de Vries-Bouwstra ${ }^{40}$, Maurizio Cutolo ${ }^{34}$ and Marta Mosca ${ }^{1,41 凶}$

${ }^{\top}$ Rheumatology Unit, Azienda Ospedaliero Universitaria Pisana, Pisa, Italy.

${ }^{2}$ Spanish Association for Antiphospholipid Syndrome (SAF España), Elche, Spain.

${ }^{3}$ Department of Rheumatology and Clinical Immunology, Charité University Medicine Berlin, Berlin, Germany.

${ }^{4}$ Department of Internal Medicine, Hospital Pitié-Salpêtrière, Assistance Publique Hôpitaux de Paris, French National Referral Center for SLE and APS, Paris, France.

${ }^{5}$ Auto-immune Disease Unit, Hospital de Curry Cabral, Centro Hospitalar de Lisboa Central, EPE,

Lisbon, Portugal.

${ }^{6}$ Service de rhumatologie, Hôpitaux Universitaires de Strasbourg, Centre National de Référence des Maladies Systémiques et Auto-immunes Rares Grand-Est Sud-Ouest (RESO), Strasbourg, France.

${ }^{7}$ Department of Allergology, Rheumatology and Clinical Immunology, University Children's Hospital, University Medical Centre Ljubljana, Ljubljana, Slovenia.

${ }^{8}$ Referral Center for Systemic Autoimmune Diseases, Fondazione IRCCS Ca' Granda Ospedale Maggiore Policlinico di Milano, Milan, Italy.

${ }^{9}$ University of Pisa, Pisa, Italy.

${ }^{10}$ Institute of Management, Scuola Superiore Sant'Anna, Pisa, Italy.

${ }^{11}$ Department of Rheumatology, IRCCS Policlinico San Matteo Foundation, Pavia, Italy.

${ }^{12}$ Service de Médecine Interne, Hôpital Cochin, Centre de Référence Maladies systémiques Autoimmunes Rares d'lle de France, Assistance Publique-Hôpitaux de Paris (AP-HP), Université de Paris, Paris, France.

${ }^{13}$ Lupus Europe, Brussels, Belgium.

${ }^{14}$ Rheumatology Unit, Department of Medicine, University of Padova, Padova, Italy.

${ }^{15}$ Medical Genetics Laboratory, Molecular Medicine Department, San Camillo Forlanini Hospital, Sapienza University, Rome, Italy.

${ }^{16}$ Department of Rheumatology, University Hospital Düsseldorf, Heinrich-Heine-University Düsseldorf, Düsseldorf, Germany.

${ }^{17}$ Rheumatology Department, Hospital de Santa Maria, Centro Hospitalar Lisboa Norte, Lisbon Academic Medical Centre, Lisbon, Portugal.

${ }^{18}$ Flemish Association for Hereditary Connective Tissue Disorders, Koersel, Belgium.

${ }^{19} \mathrm{Federation}$ of European Scleroderma Associations (FESCA), Milan, Italy.

${ }^{20}$ Deutsche Ehlers-Danlos Initiative e.V., Furth, Germany.

${ }^{21}$ Liga Portuguesa Contra as Doenças Reumáticas, Lisbon, Portugal.

${ }^{22}$ Département de Médecine Interne et Immunologie Clinique, Centre de Référence des Maladies
Systémiques et Auto-Immunes Rares du Nord-Ouest (CERAINO), LIRIC, INSERM, Univ. Lille, CHU Lille, Lille, France.

${ }^{23}$ Department of Rheumatology, Cliniques Universitaires Saint-Luc, Brussels, Belgium.

${ }^{24}$ Department of Dermatology, Universitätsklinikum, Cologne, Germany.

${ }^{25}$ Department of Rheumatology and Clinical Immunology, University Medical Center Utrecht Utrecht University, Utrecht, Netherlands.

${ }^{26}$ Center for Medical Genetics, Ghent University Hospital, Ghent, Belgium.

${ }^{27}$ Université Paris-Saclay, INSERM, CEA, Centre de recherche en Immunologie des infections virales et des maladies auto-immunes; AP-HP. Université Paris-Saclay, Hôpital Bicêtre, Rheumatology Department, Le Kremlin Bicêtre, Paris, France. ${ }^{28}$ Clinical immunology department, Hôpitaux Universitaires de Strasbourg, Centre National de Référence des Maladies Systémiques et Auto-immunes Rares Grand-Est Sud-Ouest (RESO), Strasbourg, France.

${ }^{29}$ Relapsing Polychondritis Awareness and Support, Worcester, UK.

${ }^{30}$ Division of Rheumatology and Scleroderma Unit, Department of Clinical and Experimental Medicine, AOU Careggi, University of Florence, Florence, Italy.

${ }^{31}$ Department of Rheumatology and Clinical Immunology, Kerckhoff Klinik, Justus Liebig University of Giessen, Bad Nauheim, Germany.

${ }^{32}$ Department of Rheumatology, Emergency County Teaching Hospital, Cluj-Napoca, Romania.

${ }^{33}$ Department of Rheumatology, Ghent University Hospital Department of Internal Medicine, Ghent, Belgium.

${ }^{34}$ Research Laboratory and Academic Division of Clinical Rheumatology, Department of Internal Medicine, IRCCS Polyclinic Hospital San Martino, University of Genoa, Genoa, Italy.

${ }^{35}$ National Centre for Rare Diseases, Istituto Superiore di Sanità, Rome, Italy.

${ }^{36}$ Directorate-General for Health and Food Safety, European Commission, Brussels, Belgium.

${ }^{37}$ Rheumatology and Clinical Immunology Unit, ASST-Spedali Civili and University of Brescia, Brescia, Italy.

${ }^{38}$ Department of Internal Medicine and Immunology, Erasmus MC, Rotterdam, Netherlands.

${ }^{39}$ Liga Portuguesa Contra as Doenças Reumáticas, Núcleo Síndrome de Sjögren, Lisbon, Portugal.

${ }^{\circ}$ Department of Rheumatology, Leiden University Medical Center, Leiden, The Netherlands.

${ }^{41}$ Rheumatology Unit, University of Pisa, Pisa, Italy.

凶e-mail:ern.reconnet@ao-pisa.toscana.it https://doi.org/10.1038/s41584-020-00565-z Published online 6 January 2021

1. World Health Organization. WHO Coronavirus Disease (COVID-19) Dashboard. https://covid1 19.who.int/ (2020).

2. Rare Barometer. How has COVID-19 impacted people with rare diseases? https://download2.eurordis.org/ $\mathrm{rbv} /$ covid 19survey/covid_infographics_final.pdf (2020).

3. McInnes, I. B. COVID-19 and rheumatology: first steps towards a different future? Ann. Rheum. Dis. 79, 551-552 (2020).

4. Richez, C., Lazaro, E., Lemoine, M., Truchetet, M. E. $\&$ Schaeverbeke, T. Implications of COVID-19 for the management of patients with inflammatory rheumatic diseases. Joint Bone Spine 87, 187-189 (2020).

5. Felten, R., Chatelus, E. \& Arnaud, L. How and why are rheumatologists relevant to COVID-19? Joint Bone Spine https://doi.org/10.1016/j.jbspin.2020.04.006 (2020). 
6. Mahévas, M. et al. Clinical efficacy of hydroxychloroquine in patients with COVID-19 pneumonia who require oxygen: observational comparative study using routine care data. BMJ 369 , m1844 (2020)

7. Ferro, F. et al. COVID-19: the new challenge for rheumatologists. Clin. Exp. Rheumatol. 38, 17-80 (2020).

8. Ferro, F. COVID-19: the new challenge for rheumatologists. First update. Clin. Exp. Rheumatol. 38, 373-382 (2020)

9. Yazdany, J. \& Kim, A. H. J. Use of hydroxychloroquine and chloroquine during the COVID-19 pandemic: what every clinician should know. Ann. Intern. Med. 172, 754-755 (2020)

10. Dejaco, C. et al. Influence of COVID-19 pandemic on decisions for the management of people with inflammatory rheumatic and musculoskeletal diseases: a survey among EULAR countries. Ann. Rheum. Dis. https://doi.org/10.1136/annrheumdis-2020-218697 (2020).

11. Ziadé, N. et al. The impact of COVID-19 pandemic on rheumatology practice: a cross-sectional multinational study. Clin. Rheumatol. 39, 3205-3213 (2020).

12. Misra, D. P., Agarwal, V., Gasparyan, A. Y. \& Zimba, O. Rheumatologists' perspective on coronavirus disease 19 (COVID-19) and potential therapeutic targets. Clin. Rheumatol. 39, 2055-2062 (2020).

13. Monti, S. et al. Clinical course of COVID-19 in a series of patients with chronic arthritis treated with immunosuppressive targeted therapies. Ann. Rheum Dis. 79, 667-668 (2020)

14. Yen, E., Singh, D. R. $\&$ Singh, R. R. 48-year trends in systemic sclerosis mortality, 1968-2015: a United States population-based study. Arthritis Care Res. https://doi.org/10.1002/acr.24411 (2020).

15. Ogata, Y. et al. Morbidity and mortality in antiphospholipid syndrome based on cluster analysis: a 10-year longitudinal cohort study. Rheumatology https://doi.org/10.1093/rheumatology/keaa542 (2020).

16. Chen $M$., Wei $Y$, Zhang $\mathrm{O}$., Wan $\mathrm{O}$., Chen $\mathrm{X}$. Epidemiology and clinical characteristics of COVID-19 in rheumatic diseases at a tertiary care hospital in Wuhan, China. Clin. Exp. Rheumatol. https://www.clinexprheumatol.org/article.asp?a=15961 (2020).

17. Mason, A., Rose, E. \& Edwards, C. J. Clinical management of lupus patients during the COVID-19 pandemic. Lupus 29, 1661-1672 (2020)

18. Minniti, A et al. Taking care of systemic sclerosis patients during COVID-19 pandemic: rethink the clinical activity. Clin. Rheumatol. 39, 2063-2065 (2020).

19. Robinson, P. C. \& Yazdany, J. The COVID-19 global rheumatology alliance: collecting data in a pandemic. Nat. Rev. Rheumatol. 16, 293-294 (2020).

20. Società Italiana di Reumatologia. Registro COVID-19-RMD SIR. https://redcap.reumatologia.it/ surveys/?s=YLDERDE88W (2020).

21. Gianfrancesco, M. A. et al. Rheumatic disease and COVID-19: initial data from the COVID-19 Global Rheumatology Alliance provider registries. Lancet Rheumatol. 2, e250-e253 (2020).

22. Gianfrancesco, $\mathrm{M}$. et al. Characteristics associated with hospitalisation for COVID-19 in people with rheumatic disease: data from the COVID-19 Global Rheumatology Alliance physician-reported registry. Ann. Rheum. Dis. 79, 859-866 (2020).

23. Hașlak, F., Yıldız, M., Adrovic, A., Barut, K. \& Kasapçopur, Ö. Childhood rheumatic diseases and
COVID-19 pandemic: an intriguing linkage and a new horizon. Balkan Med. J. 37, 184-188 (2020).

24. Landewé, R. B. et al. EULAR provisional recommendations for the management of rheumatic and musculoskeletal diseases in the context of SARS-CoV-2. Ann. Rheum. Dis. 79, 851-858 (2020).

25. Matucci-Cerinic, M. et al. Systemic sclerosis and the COVID-19 pandemic: World Scleroderma Foundation preliminary advice for patient management. Ann. Rheum. Dis. 79, 724-726 (2020).

26. Bozzalla Cassione, E. et al. COVID-19 infection in a northern-Italian cohort of systemic lupus erythematosus assessed by telemedicine. Ann. Rheum. Dis. 79 1382-1383 (2020)

27. Cavagna, L. et al. Telemedicine in rheumatology: a reliable approach beyond the pandemic. Rheumatology https://doi.org/10.1093/rheumatology/keaa554 (2020).

28. Romão, V. C. et al. Rheumatology practice amidst the COVID-19 pandemic: a pragmatic view. RMD Open 6 , e001314 (2020)

29. Wuyts, W. A., Cottin, V., Spagnolo, P. \& Wells, A. U. (Eds) Pulmonary Manifestations of Systemic Diseases. (European Respiratory Society, 2019).

30. Takla, M. \& Jeevaratnam, K. Chloroquine, hydroxychloroquine, and COVID-19: systematic review and narrative synthesis of efficacy and safety. Saudi Pharm J. https://doi.org/10.1016/j.jsps.2020.11.003 (2020).

31. Lupus Europe. Hydroxychloroquine: patient reported availability. A survey amongst lupus patients. https:// www.lupus-europe.org/wp-content/uploads/2020/04/ survey-Hcq-Learning-0410-Fin.pdf (2020).

32. World Health Organisation. COVID-19: Operational guidance for maintaining essential health services during an outbreak Interim guidance, 25 March 2020 https://apps.who.int/iris/bitstream/handle/10665 331561/WHO-2019-nCoV-essential health services2020.1-eng. . $d f$ ? sequence $=1$ EisAllowed $=y$ (2020).

33. Mazzoleni, S., Turchetti, G. \& Ambrosino, N. The COVID-19 outbreak: from "black swan" to global challenges and opportunities. Pulmonology 26 117-118 (2020)

34. European Commission. The common EU response to COVID-19. https://europa.eu/european-union/ coronavirus-response_en\#eu-actions (2020).

35. European Commission. ERNs and COVID-19. https:// ec.europa.eu/health/ern/covid-19 en (2020).

36. European Commission. What are the ERNs doing to help patients affected by rare diseases and COVID-19? https://ec.europa.eu/health/sites/health/files/ern/docs/ covid 19 erns en. pdf (2020).

37. ERN ReCONNET. Update on COVID-19 outbreak. https://reconnet.ern-net eu/update-on-covid-19outbreak/ (2020).

38. Fai2R. The news. https://www.fai2r.org/actualites/ covid-19 (2020).

39. ERN ReCONNE. COVID-19: ERN ReCONNET endorsed recommendations, https://reconnet.ern-net.eu/ covid-19-ERN\%20ReCONNET/ (2020).

40. Cutolo, M., Smith, V. \& Paolino, S. Understanding immune effects of oestrogens to explain the reduced morbidity and mortality in female versus male COVID-19 patients. Comparisons with autoimmunity and vaccination. Clin. Exp. Rheumatol. 38, 383-386 (2020).

41. Garazzino, S. et al. Multicentre Italian study of SARS-CoV-2 infection in children and adolescents, preliminary data as at 10 April 2020. Eurosurveillance 25, 2000600 (2020)

42. Rivieccio, B. A. et al. Heterogeneity of COVID-19 outbreak in Italy. Acta Biomed. 91, 31-34 (2020).

43. Istituto Superiore di Sanità. Special section on COVID-19. https://www.iss.it/coronavirus (2020).
44. Istituto Superiore di Sanità. Rapporti ISS COVID-19 in English https://www iss. it/rapporti-iss-covid-19in-english (2020).

Acknowledgements

This publication was funded by the European Union's Health Programme (2014-2020).

\section{Author contributions}

R.T., S.C. and D.M. researched data for the article. R.T., S.C. LC B. A.C. D M M C and M M made substantial contributions to discussion of the content. R.T., S.A., A.C., C.F., I.C., J.G., V.G., D.M., L.M., D.T., G.T., A.V. and M.M. wrote the article. R.T., S.A., T.A., Z.A., A.M.A., L.A., T.A., L.B., S.B., G.R.B. S.C., L.C., N.C.-C., A.D., A.F., R.F.-B., J.E.F., A.G., E.H., F.H., L.I., T.K M.L. FM X.M D.M TM M.M-C. A.M C.M L.M U.M.-L., S.R., V.C.R., M.S., V.S., A.S., F.T., D.T., A.V.T., E.T., A.T S.T., G.T., M.P.v.H., J.M.v.L., J.K.d.V.-B., M.C. and M.M reviewed/edited the manuscript before submission.

\section{Competing interests}

The authors declare no competing interests.

\section{Disclaimer}

ERN ReCONNET is one of the 24 European Reference Networks (ERNs) approved by the ERN Board of Member States. The ERNs are co-funded by the EC (European Commission). The content of this publication represents the views of the authors only and it is their sole responsibility; it cannot be considered to reflect the views of the EC and/or the Consumers, Health, Agriculture and Food Executive Agency (CHAFEA) or any other body of the European Union. The EC and CHAFEA do not accept any responsibility for use that may be made of the information it contains.

\section{Peer review information}

Nature Reviews Rheumatology thanks E. Price and the other, anonymous, reviewer(s) for their contribution to the peer review of this work.

\section{Publisher's note}

Springer Nature remains neutral with regard to jurisdictional claims in published maps and institutional affiliations.

Springer Nature or its licensor holds exclusive rights to this article under a publishing agreement with the author(s) or other rightsholder(s); author self-archiving of the accepted manuscript version of this article is solely governed by the terms of such publishing agreement and applicable law.

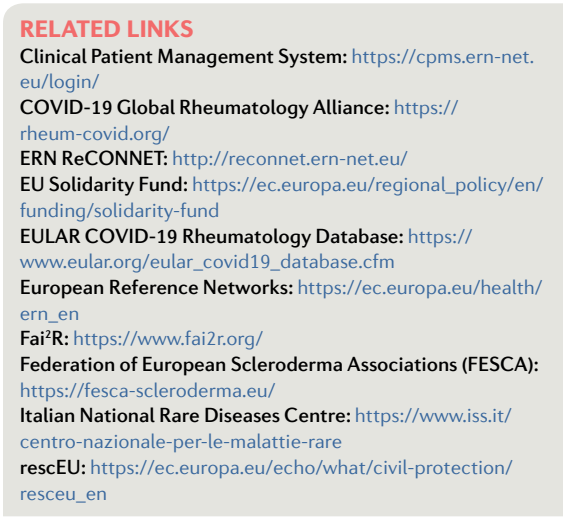

(c) Springer Nature Limited 2021, corrected publication 2022 